\title{
Proof of the Razumov-Stroganov conjecture for some infinite families of link patterns
}

\author{
P. Zinn-Justin \\ Laboratoire de Physique Théorique et Modèles Statistiques (CNRS, UMR 8626); \\ Univ Paris-Sud, F-91405 Orsay, France \\ pzinn@lptms.u-psud.fr
}

Submitted: Sep 12, 2006; Accepted: Nov 7, 2006; Published: Nov 23, 2006

AMS Subject Classifications: Primary 05A19; Secondary 52C20, 82B20

\begin{abstract}
We prove the Razumov-Stroganov conjecture relating ground state of the $O(1)$ loop model and counting of Fully Packed Loops in the case of certain types of link patterns. The main focus is on link patterns with three series of nested arches, for which we use as key ingredient of the proof a generalization of the MacMahon formula for the number of plane partitions which includes three series of parameters.
\end{abstract}

\section{Introduction}

The Razumov-Stroganov (RS) conjecture [1] relates the components of the ground state of the $O(1)$ loop model, which are indexed by link patterns (pairing of points on a circle) to the numbers of Fully Packed Loop configurations (FPL) on a square grid with a connectivity of external vertices given by the same link pattern. Despite considerable activity around this conjecture $[2,3,4,5,6,7,8]$, it has not been proved yet. It is the author's belief, however, that the work [9] was a significant step in this direction: in it, an inhomogeneous loop model was introduced in order to make the ground state a polynomial of the inhomogeneities (spectral parameters). This way, a corollary of the RS conjecture (which was already formulated in [10]), namely that the properly normalized sum of all components of the loop model ground state equals the total number of FPL, also known as the number of Alternating Sign Matrices, was proved.

The present work tries to demonstrate in a very simple setting how the methods of [9] could help to prove the RS conjecture by considering a special subset of possible link patterns, namely those with few "little arches" (arches connecting neighbors). Consider the link patterns of Fig. 1. They are made of three sets of $a, b, c$ nested arches. Here $a, b, c$ are arbitrary integers such that $a+b+c=n$ where the size of the system is $2 n$. The model depends on $2 n$ complex numbers $\alpha_{1}, \ldots, \alpha_{b+c}, \beta_{1}, \ldots, \beta_{a+c}, \gamma_{1}, \ldots, \gamma_{a+b}$ which are, up to multiplication by a power of $q$ (as will be explained below), the spectral parameters of the model. Note there are several good reasons to restrict oneself to such link patterns, a particularly obvious one being that we know the corresponding number of FPL: it was computed in [11] - and happens to be equal to the number of Plane Partitions in a hexagon of shape $a \times b \times c$ ! 


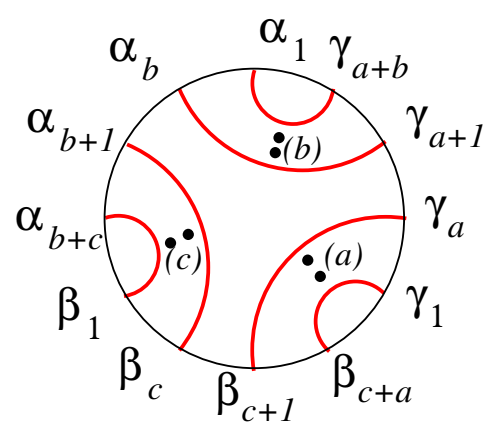

Fig. 1: Link pattern with 3 sets of nested arches.

The reader is referred to [9] for details concerning the $O(1)$ loop model. We briefly recall its definition here, if only to fix notations. Let $n$ be an integer, and consider a complex vector space equipped with a basis indexed by non-crossing link patterns: the latter are by definition pairings of $2 n$ points on a circle, in such a way that the pairings can be represented by non-crossing edges inside the circle. On this space we define the action of a linear operator, the so-called transfer matrix $T_{n}\left(t \mid z_{1}, \ldots, z_{2 n}\right)$, which depends on complex parameters $t, z_{1}, \ldots, z_{2 n}$, by the following graphical description:

$$
T_{n}\left(t \mid z_{1}, \ldots, z_{2 n}\right)=\prod_{i=1}^{2 n}\left(\frac{q z_{i}-q^{-1} t}{q t-q^{-1} z_{i}} \backslash \square+\frac{z_{i}-t}{q t-q^{-1} z_{i}} \square\right)
$$

where $q=\mathrm{e}^{2 i \pi / 3}$, and the symbolic product over $i$ means that the plaquette of index $i$ should be inserted at vertex $i$. The result is that, starting from a given link pattern, the action of $T_{n}$ produces a new link pattern by adding a circular strip of plaquettes and removing any closed loops thus created. The coefficients in Eq. (1.1), in the range of parameters where they are real, are simply probabilities of inserting the corresponding plaquettes, parametrized in a convenient way in terms of the $z_{i} / t$. As a consequence of the Yang-Baxter equation, $\left[T_{n}(t), T_{n}\left(t^{\prime}\right)\right]=0$ with all other parameters $z_{i}$ fixed.

Since $T_{n}$ is a stochastic matrix, it has the obvious left eigenvector $(1, \ldots, 1)$ with eigenvalue 1 . Therefore, it also has a corresponding right eigenvector, which is unique for generic values of the $z_{i}$ :

$$
T_{n}\left(t \mid z_{1}, \ldots, z_{2 n}\right) \Psi_{n}\left(z_{1}, \ldots, z_{2 n}\right)=\Psi_{n}\left(z_{1}, \ldots, z_{2 n}\right)
$$

One can normalize $\Psi_{n}$ in such a way that its components $\Psi_{n, \pi}$ in the basis of link patterns $\pi$ are coprime polynomials of the $z_{i}$. One still has an arbitrary numerical constant in the normalization of $\Psi_{n}$. Consider now the homogeneous limit when all $z_{i}$ equal 1 . If one chooses this constant so that the smallest entry is 1 , then the $\Psi_{n, \pi}(1, \ldots, 1)$ are the subject of various conjectures, including the remarkable Razumov-Stroganov conjecture already mentioned above, that identifies them with a certain FPL enumeration problem. In what follows we shall choose another numerical normalization which is more convenient for intermediate calculations. 
In Sect. 2, we derive the main formula for the entries of the ground state of the $O(1)$ loop model corresponding to link patterns with three sets of nested arches (Fig. 1). In Sect. 3 we establish the connection with plane partitions (or dimers). Sect. 4 discusses the (partial or total) homogeneous limit. Sect. 5 briefly describes the extension to more general link patterns for which the corresponding enumeration of FPL is known. Sect. 6 concludes.

\section{Recurrence relations and their solution}

In [9], a certain number of relations were shown to be satisfied by $\Psi_{n}$, the ground state eigenvector of the $O(1)$ loop model of size $2 n$. We shall need the following three facts:

THEOREM 4 OF [9]. The components of $\Psi_{n}$ are homogeneous polynomials of total degree $n(n-1)$, and of partial degree at most $n-1$ in each variable $z_{i}$.

TheOREM 1 of [9]. The entries $\Psi_{n, \pi}$ of the groundstate eigenvector satisfy:

$$
\Psi_{n, \pi}\left(z_{1}, \ldots, z_{2 n}\right)=\left(\prod_{s \in E_{\pi}} \prod_{\substack{i, j \in s \\ i<j}}\left(q z_{i}-q^{-1} z_{j}\right)\right) \Phi_{n, \pi}\left(z_{1}, \ldots, z_{2 n}\right)
$$

where $\Phi_{n, \pi}$ is a polynomial which is symmetric in the set of variables $\left\{z_{i}, i \in s\right\}$ for each $s \in E_{\pi}$, and $E_{\pi}$ is the partition of $\{1, \ldots, 2 n\}$ into maximal sequences of consecutive points not connected to each other by arches of $\pi$.

THEOREM 3 OF [9]. If two neighboring parameters $z_{i}$ and $z_{i+1}$ are such that $z_{i+1}=q^{2} z_{i}$, then either of the two following situations occur for the components $\Psi_{n, \pi}$ :

(i) the pattern $\pi$ has no arch joining $i$ to $i+1$, in which case according to Theorem 1 ,

$$
\Psi_{n, \pi}\left(z_{1}, \ldots, z_{i}, z_{i+1}=q^{2} z_{i}, \ldots, z_{2 n}\right)=0
$$

(ii) the pattern $\pi$ has a little arch joining $i$ to $i+1$, in which case

$$
\begin{aligned}
& \Psi_{n, \pi}\left(z_{1}, \ldots, z_{i}, z_{i+1}=q^{2} z_{i}, \ldots, z_{2 n}\right)= \\
& \left(\prod_{\substack{k=1 \\
k \neq i, i+1}}^{2 n}\left(q z_{i}-z_{k}\right)\right) \Psi_{n-1, \pi^{\prime}}\left(z_{1}, \ldots, z_{i-1}, z_{i+2}, \ldots, z_{2 n}\right)
\end{aligned}
$$

where $\pi^{\prime}$ is the link pattern $\pi$ with the little arch $i, i+1$ removed.

In what follows we shall concentrate on components corresponding to link patterns with three sets of nested arches of size $a, b, c$, which we shall denote by $\Psi_{a, b, c}$. The 
spectral parameters are relabelled as $z_{i}=\alpha_{i}, q \beta_{i}, q^{2} \gamma_{i}$ according to the pattern of Fig. 1. Thanks to Theorem 1, we can write

$$
\Psi_{a, b, c}=\prod_{1 \leq i<j \leq b+c}\left(q \alpha_{i}-q^{-1} \alpha_{j}\right) \prod_{1 \leq i<j \leq a+c}\left(q \beta_{i}-q^{-1} \beta_{j}\right) \prod_{1 \leq i<j \leq a+b}\left(q \gamma_{i}-q^{-1} \gamma_{j}\right) \Phi_{a, b, c}
$$

where the arguments $\left\{z_{i}\right\}=\left\{\alpha_{i}, q \beta_{i}, q^{2} \gamma_{i}\right\}$ have been suppressed for brevity. According to Theorems 1 and $4, \Phi_{a, b, c}$ is a polynomial of total degree $a b+b c+c a$, and a symmetric polynomial of the $\left\{\alpha_{i}\right\}$ of degree at most $a$ in each, of the $\left\{\beta_{i}\right\}$ of degree at most $b$ in each, and of the $\left\{\gamma_{i}\right\}$ of degree at most $c$ in each.

We now rewrite Eq. (2.3) in the case when $z_{i}$ is the last parameter $\alpha$ and $z_{i+1}$ is the first parameter $\beta$, in terms of $\Phi_{a, b, c}$. Since the latter is a symmetric function it is actually irrelevant which $\alpha$ and which $\beta$ are singled out, and the result is:

$$
\left.\Phi_{a, b, c}\right|_{\beta_{j}=\alpha_{i}}=\prod_{k=1}^{a+b}\left(\alpha_{i}-\gamma_{k}\right) \Phi_{a, b, c-1}
$$

where the parameters of $\Phi_{a, b, c-1}$ are the same as those of $\Phi_{a, b, c}$, except $\alpha_{j}$ and $\beta_{i}$ are removed. Since $\Phi_{a, b, c}$ is of degree $a$ in $\alpha_{i}$, the equations $(2.5)$ with $j=1, \ldots, a+c$ and fixed $i$ determine entirely $\Phi_{a, b, c}$ as long as $c \geq 1$. They form a very simple recurrence relation which is supplemented by the initial condition $\Phi_{a, b, 0}$ : this corresponds to the so-called "base link pattern" (Fig. 2), which is entirely factorized by Theorem 1:

$$
\Phi_{a, b, 0}=\prod_{i=1}^{b} \prod_{j=1}^{a}\left(\alpha_{i}-\beta_{j}\right)
$$

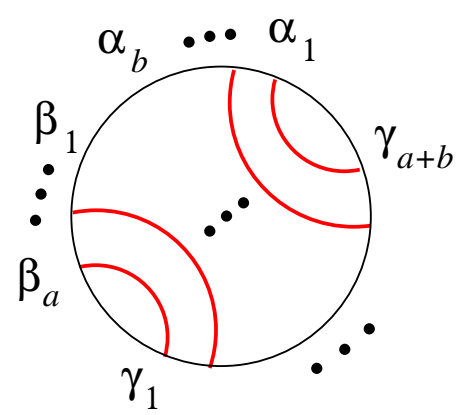

Fig. 2: Base link pattern.

We now claim that the following Ansatz solves the recurrence relation:

$$
\Phi_{a, b, c}=\sum_{\substack{I \subset\{1, \ldots, b+c\} \\ \# I=c}} \frac{\prod_{i \notin I} \prod_{j=1}^{a+c}\left(\alpha_{i}-\beta_{j}\right) \prod_{i \in I} \prod_{k=1}^{a+b}\left(\alpha_{i}-\gamma_{k}\right)}{\prod_{i \in I} \prod_{j \notin I}\left(\alpha_{i}-\alpha_{j}\right)}
$$


The case $c=0$ forces $I=\emptyset$ and we recover immediately Eq. (2.6). Next, notice that the expression of Eq. (2.7) is symmetric in all three sets of variables: it is obvious for the $\left\{\beta_{i}\right\}$ and the $\left\{\gamma_{i}\right\}$; it is also clear for the $\left\{\alpha_{i}\right\}$ since the summation over all possible subsets of cardinality $c$ is invariant by permutation of $\{1, \ldots, b+c\}$. We can therefore choose one $\alpha_{i}$ and one $\beta_{j}$ and set them equal, say $\beta_{a+c}=\alpha_{b+c}$. This forces $b+c \in I$ in Eq. (2.7). Define $I^{\prime}=I-\{b+c\}$. The summation over $I$ can then be replaced with the summation over $I^{\prime} \subset\{1, \ldots, b+c-1\}$, and it is easy to check that cancellations in numerator and denominator reproduce Eq. (2.7) with $c \rightarrow c-1$ and the parameters $\alpha_{b+c}, \beta_{a+c}$ removed. This concludes the recurrence.

Note immediately the symmetry between the sets of variables $\left\{\beta_{i}\right\}$ and $\left\{\gamma_{i}\right\}$ in Eq. (2.7): indeed, replacing $I$ with its complement $\bar{I}$ exchanges their roles (as well as $b$ and $c$ ). However, the $\left\{\alpha_{i}\right\}$ seem to play a different role. We shall now produce an equivalent expression which restores the symmetry $\alpha \leftrightarrow \beta$, at the expense of breaking the symmetry $\beta \leftrightarrow \gamma$ :

$$
\begin{aligned}
\Phi_{a, b, c}=\frac{1}{c !} \prod_{i=1}^{b+c} \prod_{j=1}^{a+c}\left(\alpha_{i}-\beta_{j}\right) \oint & \ldots \oint_{\left[\alpha_{i}\right]} \frac{\mathrm{d} z_{1}}{2 \pi i} \cdots \frac{\mathrm{d} z_{c}}{2 \pi i} \prod_{1 \leq i<j \leq c}\left(z_{i}-z_{j}\right)^{2} \\
& \frac{\prod_{\ell=1}^{c} \prod_{k=1}^{a+b}\left(z_{\ell}-\gamma_{k}\right)}{\prod_{\ell=1}^{c} \prod_{j=1}^{a+c}\left(z_{\ell}-\beta_{j}\right) \prod_{\ell=1}^{c} \prod_{i=1}^{b+c}\left(z_{\ell}-\alpha_{i}\right)}
\end{aligned}
$$

The $c$ contour integrals $[\alpha]$ should be defined in such a way as to encircle (counterclockwise) all the poles $\alpha_{i}$ (but none of the $\beta_{j}$ ). One goes back to Eq. (2.7) by applying the Cauchy formula. Each $z_{i}$ must be evaluated at a certain $\alpha_{I_{i}}$ with $1 \leq I_{i} \leq b+c$; furthermore, the factors $\prod\left(z_{i}-z_{j}\right)$ force the $I_{i}$ to be distinct, and we reproduce after various cancellations the summation over $I=\left\{I_{1}, \ldots, I_{b+c}\right\}$ of Eq. (2.7).

The formula (2.8) is of the form of a matrix integral: the contour integral makes it essentially similar to the unitary matrix integral. This analogy will be pursued below. For now, we use a standard trick in random matrix theory, which is to introduce the Vandermonde determinant $\Delta\left(z_{i}\right)=\prod_{i<j}\left(z_{i}-z_{j}\right)=\operatorname{det}_{1 \leq i \leq c}\left(z_{i}^{j-1}\right)$, and then to note that $\operatorname{det}\left(P_{i}\left(z_{j}\right)\right)=\Delta\left(z_{i}\right) \operatorname{det} P$ where the $P_{i}, 1 \leq i \leq c$, are arbitrary polynomials of degree less than $c$ and $P$ is the $c \times c$ matrix of coefficients of the $P_{i}$. In Eq. (2.8) we have a squared Vandermonde determinant, so we can introduce another similar set of polynomials $Q_{i}$. Moving the determinants out of the integrals, we find:

$\Phi_{a, b, c}=\frac{\prod_{i=1}^{b+c} \prod_{j=1}^{a+c}\left(\alpha_{i}-\beta_{j}\right)}{\operatorname{det} P \operatorname{det} Q} \operatorname{det}_{1 \leq \ell, m \leq c}\left[\oint \frac{\mathrm{d} z}{2 \pi i} P_{\ell}(z) Q_{m}(z) \frac{\prod_{k=1}^{a+b}\left(z-\gamma_{k}\right)}{\prod_{j=1}^{a+c}\left(z-\beta_{j}\right) \prod_{i=1}^{b+c}\left(z-\alpha_{i}\right)}\right]$

In what follows, we shall be naturally led to a choice of polynomials $P$ and $Q$.

\section{Connection with plane partitions}

We now introduce a model of weighted Plane Partitions - in more physical terms, it is a model of dimers on the hexagonal lattice, but we shall prefer the language of 
Plane Partitions in what follows. Configurations are defined as tilings with lozenges of a hexagon of size $a \times b \times c$. Lozenges come in three orientations since they are made of two adjacent equilateral triangles of a regular triangular lattice. The model comes with three series of parameters $\alpha_{i}, \beta_{j}, \gamma_{k}$ living on the lines of the underlying medial Kagome lattice, see Fig. 3 (i). To each lozenge of the plane partition (or equivalently to each dimer) is associated a local Boltzmann weight $\alpha_{i}-\beta_{j}, \gamma_{k}-\beta_{j}, \alpha_{i}-\gamma_{k}$ given by the difference of the parameters of the lines crossing at its center. Note that there are exactly $a b, b c, c a$ lozenges of each orientation.

(i)

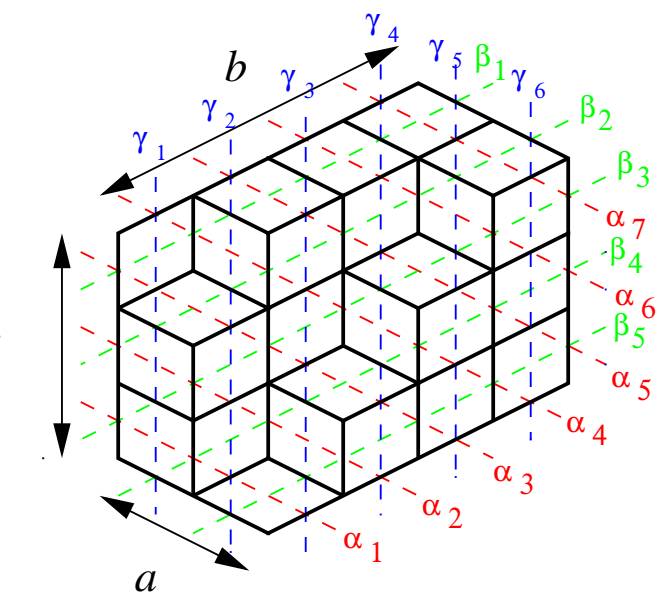

(ii)

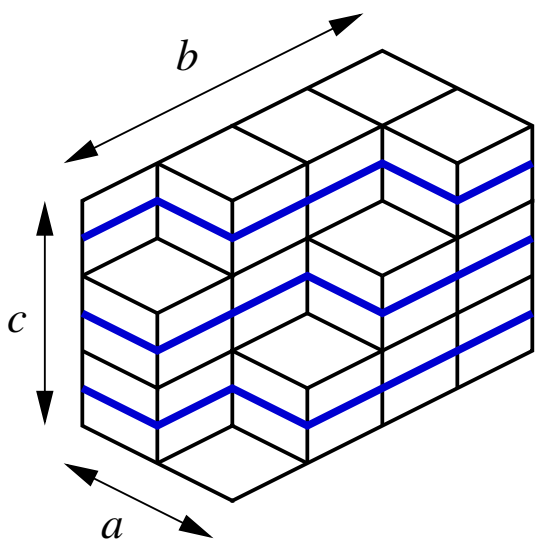

Fig. 3: (i) Plane partition and its parameters, $a=2, b=4, c=3$. (ii) Associated non-intersecting paths.

We wish to compute the partition function $Z_{a, b, c}$, i.e. the sum over all configurations of the product of local Boltzmann weights. In order to do so, it is convenient to use yet another representation in terms of non-intersecting paths (NIPs). To each plane partition one can associate $c$ paths originating from one of the sides of length $c$ and ending at the other, see Fig. 3 (ii), which simply follow two types of tiles out of the three.

One can replace the local Boltzmann weights of the tiles with a local probability for the path to go left or right, by factoring out all the possible weights of the third type of tile:

$$
Z_{a, b, c}=\prod_{\substack{1 \leq i \leq b+c, 1 \leq j \leq a+c \\ i+j>c, i+j \leq a+b+c}}\left(\alpha_{i}-\beta_{j}\right) F_{a, b ; c}
$$

and then introducing an inverse weight when the tiles of the third type are absent (i.e. where the paths are):

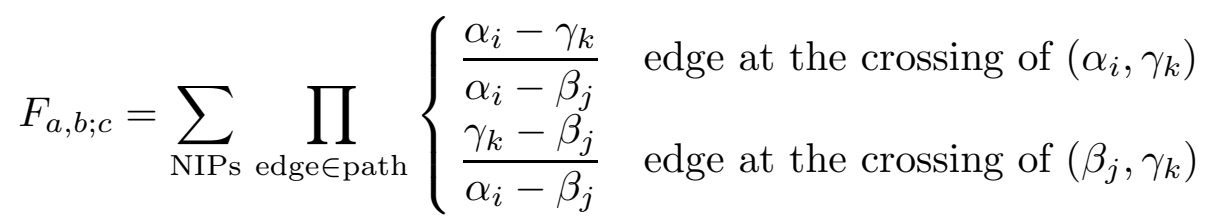


where the two orientations of the edges (or of the underlying dimers, or lozenges) determine which weight to use, and the third coordinate is given by $i+j=k+c$. These NIPs move exactly $a$ steps in one direction and $b$ steps in the other.

NIPs are free fermions, and therefore their propagator $F_{a, b ; c}$ is a determinant of one-particle propagators:

$$
F_{a, b ; c}=\operatorname{det}_{1 \leq \ell, m \leq c}[\ell \rightarrow m \text { on }(a, b, c)]
$$

where $\ell \rightarrow m$ means the probability (for a single path) to go from position $\ell$ on one side of length $c$ to position $m$ on the other. This is also known as the Lindström-GesselViennot formula [12]. The one-particle propagator is nothing but the case $c=1$, with appropriately shifted $a, b, \alpha_{i}, \beta_{j}$ :

$$
\left.[\ell \rightarrow m \text { on }(a, b, c)]=F_{a+\ell-m, b+m-\ell, 1}\left(\alpha_{\ell} \ldots \alpha_{b+m} ; \beta_{c+1-\ell} \ldots \beta_{a+c+1-m} ; \gamma_{1} \ldots \gamma_{a+b}\right)\right)
$$

We are finally led to a simple problem of computing the weighted enumeration of a single path. The following formula holds:

$$
\begin{aligned}
F_{a, b ; 1}\left(\alpha_{1}, \ldots,\right. & \left.\alpha_{b+1} ; \beta_{1}, \ldots, \beta_{a+1} ; \gamma_{1}, \ldots, \gamma_{a+b}\right) \\
& =\left(\alpha_{b+1}-\beta_{a+1}\right) \oint \frac{\mathrm{d} z}{2 \pi i} \frac{\prod_{k=1}^{a+b}\left(z-\gamma_{k}\right)}{\prod_{i=1}^{b+1}\left(z-\alpha_{i}\right) \prod_{j=1}^{a+1}\left(z-\beta_{j}\right)}
\end{aligned}
$$

where once again the contour integral encircles clockwise the $\alpha_{i}$ but not the $\beta_{i}$. This can be proved by noting that $F_{a, b ; 1}$ satisfies the following simple recurrence formula:

$$
F_{a, b ; 1}=\frac{\alpha_{b+1}-\gamma_{a+b}}{\alpha_{b+1}-\beta_{a}} F_{a-1, b ; 1}+\frac{\gamma_{a+b}-\beta_{a+1}}{\alpha_{b}-\beta_{a+1}} F_{a, b-1 ; 1}
$$

and by using $z-\gamma_{a+b}=\frac{\alpha_{b+1}-\gamma_{a+b}}{\alpha_{b+1}-\beta_{a+1}}\left(z-\beta_{a+1}\right)+\frac{\gamma_{a+b}-\beta_{a+1}}{\alpha_{b+1}-\beta_{a+1}}\left(z-\alpha_{b+1}\right)$.

Putting together Eqs. (3.1)-(3.5), one obtains

$$
Z_{a, b, c}=\prod_{\substack{1 \leq i \leq b+c, 1 \leq j \leq a+c \\ i+j>c, i+j \leq a+b+c+1}}\left(\alpha_{i}-\beta_{j}\right) \operatorname{det}_{\substack{1 \leq \ell, m \leq c \\ i+j}}\left[\oint \frac{\mathrm{d} z}{2 \pi i} \frac{\prod_{k=1}^{a+b}\left(z-\gamma_{k}\right)}{\prod_{i=\ell}^{b+m}\left(z-\alpha_{i}\right) \prod_{j=c+1-\ell}^{a+c+1-m}\left(z-\beta_{j}\right)}\right]
$$

To connect with Eq. (2.9), set

$$
\begin{aligned}
P_{\ell}(z) & =\left(z-\alpha_{1}\right) \cdots\left(z-\alpha_{\ell-1}\right)\left(z-\beta_{1}\right) \cdots\left(z-\beta_{c-\ell}\right) \\
Q_{m}(z) & =\left(z-\alpha_{b+m+1}\right) \cdots\left(z-\alpha_{b+c}\right)\left(z-\beta_{a+c+2-m}\right) \cdots\left(z-\beta_{b+c}\right)
\end{aligned}
$$

By factor exhaustion one finds immediately that $\operatorname{det} P=\prod_{i+j \leq c}\left(\alpha_{i}-\beta_{j}\right)$ and $\operatorname{det} Q=$ $\prod_{i+j \geq a+b+c+2}\left(\alpha_{i}-\beta_{j}\right)$. Plugging this into Eq. (2.9) reproduces exactly Eq. (3.7). 
We conclude that $Z_{a, b, c}=\Phi_{a, b, c}$. We have thus obtained a direct, exact relation between the components of the inhomogeneous $O(1)$ loop model corresponding to three sets of nested arches $(a, b, c)$ and the partition function of weighted plane partitions on a hexagon $a \times b \times c$.

The Razumov-Stroganov conjecture [1] claims that in the homogeneous $O(1)$ loop model, $\Psi_{a, b, c} / \Psi_{n, \min }$ (where $\Psi_{n, \min }$ is the smallest component of $\Psi_{n}$ ) must be equal to the number of Fully Packed Loop configurations (FPL) with the corresponding connectivity $(a, b, c)$. With our normalization conventions, $\Psi_{n, \min }=3^{n(n-1) / 2}$ and $\Psi_{a, b, c}=3^{\frac{(b+c)(b+c-1)+(a+c)(a+c-1)+(a+b)(a+b-1)}{4}} Z_{a, b, c}$ and $Z_{a, b, c}$ is the sum with all weights equal to $3 \frac{a b+c a+b c}{2}$, so that all factors of 3 cancel out and $\Psi_{a, b, c} / \Psi_{n, \min }$ is simply the number of plane partitions in the hexagon $a \times b \times c$. But according to [11], the number of FPLs with connectivity $(a, b, c)$ is the very same number. This proves the RS conjecture for the case of these link patterns.

\section{Relation to unitary matrix integrals and Schur functions}

Here we discuss some relations of our formulae to known objects. We use an important property of the matrix integral-like expression (2.8): it is preserved by homographic transformations.

Let us choose some of the variables to be equal, say $\alpha_{i}=\alpha$. We define $w=\frac{1}{\epsilon}(z-\alpha)$ with some $\epsilon$ such that $|\epsilon|<\min \left|\beta_{j}-\alpha\right|$ and obtain

$\Phi_{a, b, c}=\frac{\epsilon^{-c^{2}}}{c !} \oint \cdots \oint_{[0]} \prod_{\ell=1}^{c} \frac{\mathrm{d} w_{\ell}}{2 \pi i w_{\ell}} \prod_{1 \leq i<j \leq c} \Delta\left(w_{i}\right) \Delta\left(w_{i}^{-1}\right) \frac{\prod_{\ell=1}^{c} \prod_{k=1}^{a+b}\left(w_{\ell}-\frac{\gamma_{k}-\alpha}{\epsilon}\right)}{\prod_{\ell=1}^{c} \prod_{j=1}^{a+c}\left(w_{\ell}-\frac{\beta_{j}-\alpha}{\epsilon}\right) \prod_{\ell=1}^{c} w_{\ell}^{b}}$

The $w_{i}$ are integrated on contours surrounding 0 , for example $\left|w_{i}\right|=1$, so as to catch the poles at zero only. We recognize in Eq. (4.1) the usual form of a matrix integral over the unitary group $U(c)$ once angular variables are integrated out and only the eigenvalues $w_{i}$ are left. Thus,

$$
\Phi_{a, b, c}=\kappa \epsilon^{-b c} \int_{U(c)} \mathrm{d} \Omega \frac{\operatorname{det}(1+\Gamma \otimes \Omega)}{\operatorname{det}(1-\mathrm{B} \otimes \Omega)}\left(\operatorname{det} \Omega^{-1}\right)^{b}
$$

where $\kappa=\left(\frac{\prod_{k=1}^{a+b}\left(\gamma_{k}-\alpha\right)}{\prod_{j=1}^{a+c}\left(\beta_{j}-\alpha\right)}\right)^{c}, \Gamma$ is the $(a+b) \times(a+b)$ diagonal matrix with eigenvalues $\left(-\gamma_{k}+\alpha\right)^{-1}$, and $\mathrm{B}$ is the $(a+c) \times(a+c)$ diagonal matrix with eigenvalues $\left(\beta_{j}-\alpha\right)^{-1}$.

One can expand using the identities $\operatorname{det}^{-1}(1-\mathrm{B} \otimes \Omega)=\sum_{\lambda} s_{\lambda}(\Omega) s_{\lambda}(\mathrm{B})$ and $\operatorname{det}(1+$ $\Gamma \otimes \Omega)=\sum_{\lambda} s_{\lambda}(\Omega) s_{\lambda^{T}}(\Gamma)$, where $\lambda$ is a partition or Young diagram, $s_{\lambda}$ is the associated $G L$ character (with the convention that it is zero if the Young diagram has more rows than the size of the matrix), and $\lambda^{T}$ is the transposed Young diagram. Orthogonality of characters results in the simple formula

$$
\Phi_{a, b, c}=\kappa \sum_{\lambda, \mu \subset b \times c} L R_{\lambda, \mu}^{c \times b} s_{\lambda}(\mathrm{B}) s_{\mu^{T}}(\Gamma)=\kappa s_{c \times b}(\mathrm{~B} ; \Gamma)
$$


where $c \times b$ denotes the rectangular Young diagram with $c$ rows and $b$ columns, and $L R$ denotes the Littlewood-Richardon coefficients. $s_{c \times b}(\mathrm{~B} ; \Gamma)=s_{b \times c}(\Gamma ; \mathrm{B})$ is the supersymmetric Schur function, which in the case of a rectangular Young diagram is the same as a double, or factorial, Schur function. Alternatively, note that one can derive the Giambelli determinant identity for double Schur functions starting from Eq. (4.1) and pulling the determinants out of the integrals. In terms of non-intersecting lattice paths this Giambelli identity is nothing but another form of the LGV formula (and the SSYT formula for double Schur functions is simply the summation over NILPs).

If we set two sets of variables to be equal, say $\alpha_{i}=\alpha$ and $\beta_{i}=\beta$, we can define $w=\frac{z-\alpha}{z-\beta}$ to send $(\alpha, \beta)$ to $(0, \infty)$ and obtain

$$
\Phi_{a, b, c}=\frac{\operatorname{cst}}{c !} \oint \cdots \oint \frac{\mathrm{d} w_{1}}{2 \pi i} \ldots \frac{\mathrm{d} w_{c}}{2 \pi i} \prod_{1 \leq i<j \leq c} \Delta\left(w_{i}\right) \Delta\left(w_{i}^{-1}\right) \frac{\prod_{\ell=1}^{c} \prod_{k=1}^{a+b}\left(w_{\ell}-\frac{\gamma_{k}-\alpha}{\gamma_{k}-\beta}\right)}{\prod_{\ell=1}^{c} w_{\ell}^{b}}
$$

and analogously rewrite this as either a Giambelli identity or a unitary matrix integral, resulting in

$$
\Phi_{a, b, c}=\kappa^{\prime} s_{b \times c}\left(\Gamma^{\prime}\right)
$$

where $\kappa^{\prime}=(\alpha-\beta)^{a b} \prod_{k=1}^{a+b}\left(\gamma_{k}-\alpha\right)^{c}$ and $\Gamma^{\prime}$ is the $(a+b) \times(a+b)$ diagonal matrix with eigenvalues $\frac{\gamma_{k}-\beta}{\alpha-\gamma_{k}}$,

As a final check, we consider the homogeneous situation where all $z_{i}$ are equal to 1 , that is $\alpha_{i}=\alpha=1, \beta_{j}=\beta=q^{2}, \gamma_{k}=\gamma=q$. In this case $\Gamma^{\prime}=-q 1$ in Eq. (4.5). We find that $\Phi_{a, b, c}$ becomes $3^{(a b+b c+c a) / 2}$ times the dimension of the $G L(a+b)$ representation with rectangular Young diagram $b \times c$. The latter is one of the many formulae for the number of plane partitions.

\section{Generalization to four little arches}

Let us first reobtain the result of Sect. 3 in a more synthetic way. We use the fact, proved in appendix A, that when one switches the two spectral parameters of neighboring parallel lines, the partition function of plane partitions with an arbitrary geometry is unchanged. This implies that the partition function $Z_{a, b, c}$ for plane partitions introduced in Sect. 3 is a symmetric function of the spectral parameters $\left\{\alpha_{i}\right\},\left\{\beta_{j}\right\},\left\{\gamma_{k}\right\}$. At this stage, one can skip the entire reinterpretation in terms of free fermions and prove directly that it satisfies the same recurrence relations as $\Phi_{a, b, c}$. Indeed, setting say $\alpha_{1}=\beta_{a+c}$ forbids the lozenge parallel to sides $a$ and $b$ in the corner, see Fig. 4 , and thus creates two rows of "frozen" lozenges which lead us back to the case $a \times b \times(c-1)$. This provides a nice graphical interpretation of the recurrence relations (Eq. (2.5)), very much in the spirit of the recurrence relations of Korepin for the six-vertex model with Domain Wall Boundary Conditions [13]. 


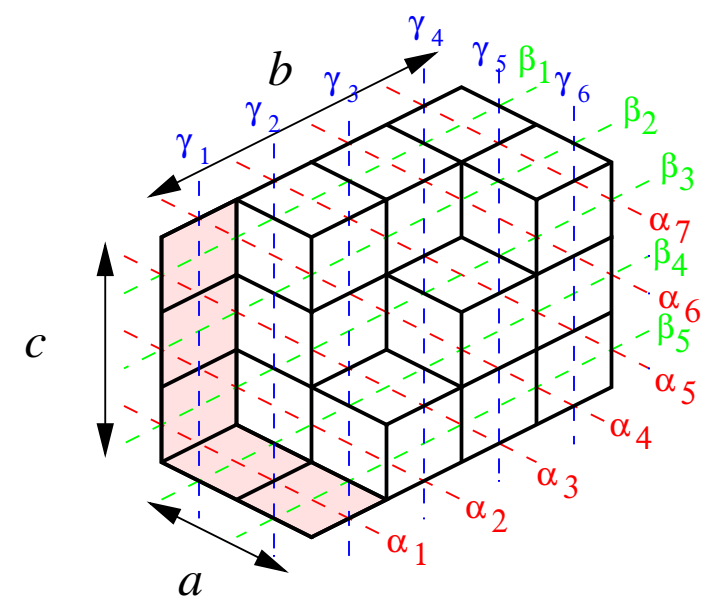

Fig. 4: Plane partition in which the $\alpha_{1}$ and $\beta_{a+c}$ rows are frozen.

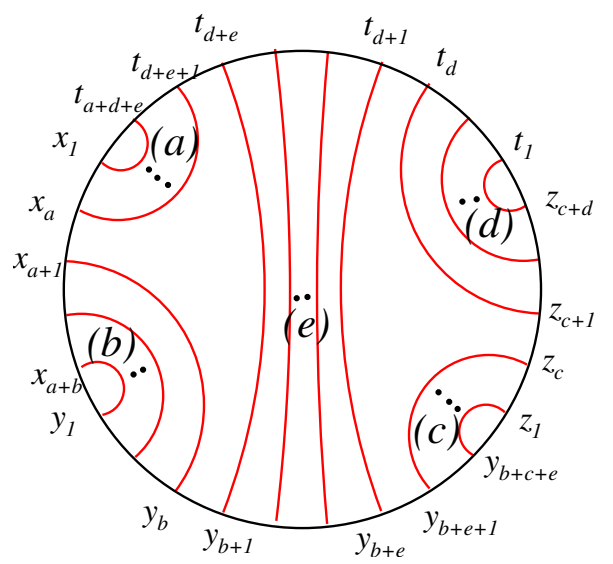

Fig. 5: Link pattern with four little arches $(a, b|e| c, d)$.

Consider now the most general link pattern which possesses four "little arches" (arches connecting neighbors), as described by Fig. 5. This is the most general family of link patterns for which FPL enumeration is known (see [14], containing as special cases the enumerations of [9] and [15]). It was shown in [14] that their enumeration is equivalent to that of certain lozenge tilings of a region of the plane with identifications, see Fig. 6 (if needed we switch $(a, b)$ and $(c, d)$ so that $b \geq d$ ). Note that there are exactly $d$ "dents" in the two identified sides of length $c+d$. Inspired by the case of three little arches, it is natural to introduce spectral parameters into the lozenge tilings as described on Fig. 6. The weight of a lozenge is equal to $q u-q^{-1} v$ where $u$ and $v$ are the spectral parameters crossing at the center of the lozenge in such a way that the line of $v$ forms an angle of $+\pi / 3$ with that of $u$ (contrary to the case of three little arches, we cannot get rid of the factors of $q$ by a redefinition of the spectral parameters). One checks that this produces a partition function which has degree at most: $c+d+e$ in each $x_{i}, a+d$ in each $y_{i}, a+b+e$ in each $z_{i}, b+c$ in each $t_{i}$, as should be. As a 


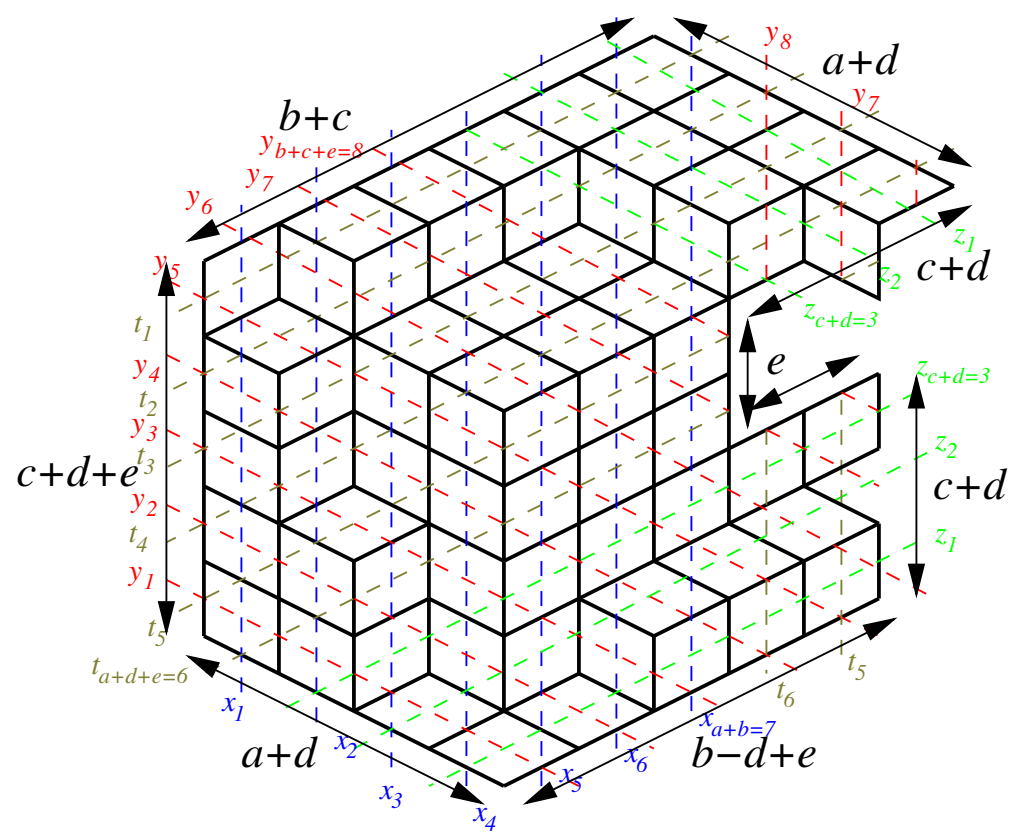

Fig. 6: Lozenge tiling corresponding to the link pattern $(3,4|2| 2,1)$ and its parameterization. The sides of length $c+d$ are identified.

consequence of Appendix A, it is symmetric in each set of variables.

It is now easy to check that these partition functions satisfy all the required recurrence relations. Among the various possibilities, two are depicted on Fig. 7. If one sets $t_{1}=q^{2} x_{1}$, the first rows of the sides of length $a+d$ and $c+d+e$ are frozen, and once these are removed one obtains the tiling with $a \rightarrow a-1$. Similarly, if $t_{1}=q^{-2} z_{1}$, the first rows of the sides of lengths $b+c$ and $a+d$ are frozen, and one dent becomes locked in first position. Once these rows are removed one recovers the tiling with $d \rightarrow d-1$ (with, in particular, one less dent).
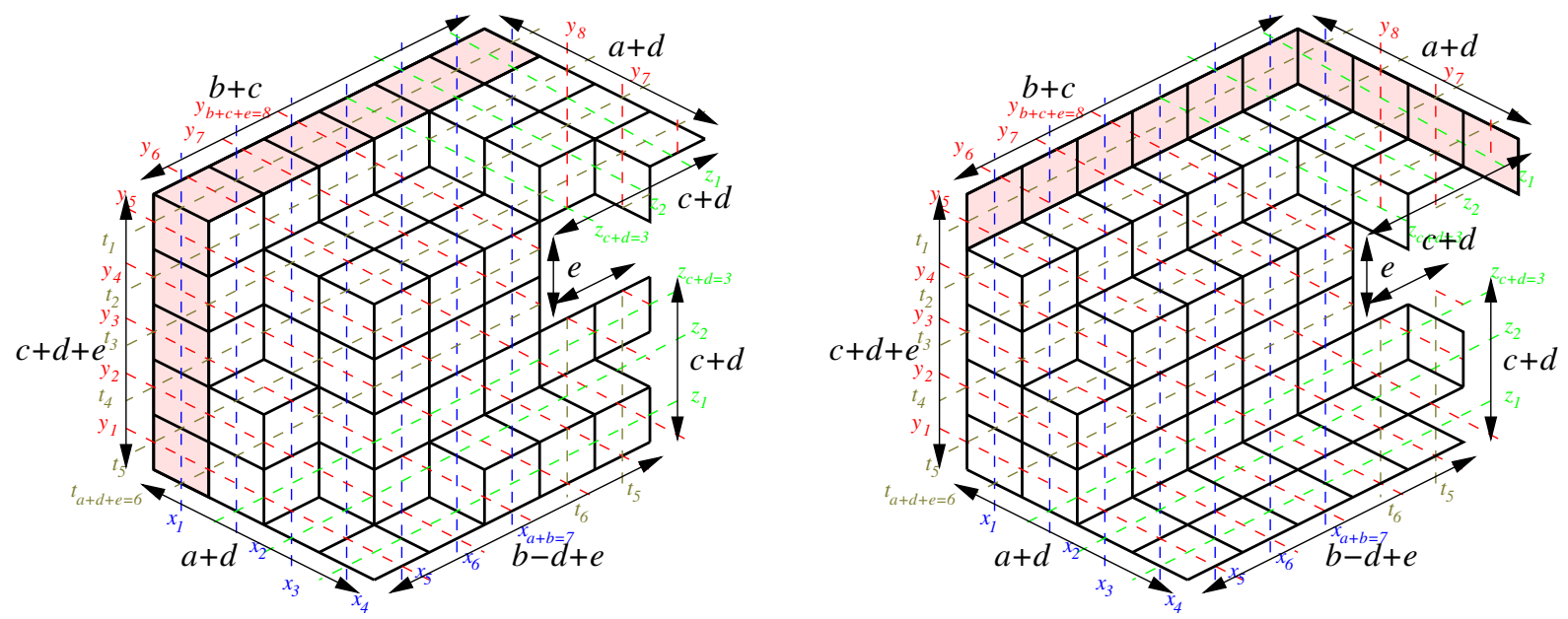

Fig. 7: Lozenge tilings with $\left(t_{1}, x_{1}\right)$ and $\left(t_{1}, z_{1}\right)$ rows frozen. 
Since the partition function is of degree $b+c$ in $t_{1}$ and is known at $a+b+c+d$ values of $t_{1}$, it is entirely fixed. The recurrence allows us to reach either $d=0$ or $a=0$, at which point, up to an additional frozen region, one recovers the tiling of a hexagon, a case which has already been treated. Now the component of $\Psi_{n}$ corresponding to the link pattern $(a, b|e| c, d)$, once rid of its factors $\prod_{i<j}\left(q x_{i}-q^{-1} x_{j}\right) \prod_{i<j}\left(q y_{i}-q^{-1} y_{j}\right) \prod_{i<j}\left(q z_{i}-\right.$

$\left.q^{-1} z_{j}\right) \prod_{i<j}\left(q t_{i}-q^{-1} t_{j}\right)$, satisfies the very same recurrence relations (of course at each step one must check that the prefactors in the recurrence match); therefore it is equal to the partition function of lozenge tilings described above. In particular, when all spectral parameters are equal to 1 , the homogeneous component is equal to the number of such tilings, as predicted by the RS conjecture.

\section{Conclusion}

In this article, we have proved in detail how the component $\Psi_{a, b, c}$ of the ground state of the $O(1)$ loop model corresponding to the link pattern with three series of nested arches $(a, b, c)$, once properly normalized, is equal to the number of plane partitions in a hexagon of size $a \times b \times c$. This is a highly non-trivial check of the Razumov-Stroganov conjecture since these link patterns form an infinite series. We have briefly described how the proof can be extended to more general link patterns, $(a, b|e| c, d)$.

In fact, we have found more than this: just as in [9] the sum of components was actually computed for arbitrary spectral parameters, here we have found that $\Psi_{a, b, c}$ with spectral parameters is the partition function of plane partitions with some local Boltzmann weights. This might seem unsurprising since one can hope that there is a unique "natural" way to introduce spectral parameters into the model; however, if ones tries to connect to FPL configurations, in which the RS conjecture is formulated, one is faced with a subtle problem: how to map back the plane partitions onto the square lattice of the FPL in such a way as to make sense of the spectral parameter dependence? Answering this is probably related to proving the full RS conjecture. We hope to come back to this point in the future.

More obvious extensions of this work should be mentioned. Firstly, the formulae of [9] allow to obtain mechanically more ground state components by considering local modifications of the ones presented here. It would be interesting to see if this computation of ground state components gives us any hint on the corresponding FPL enumerations, which are in general not known.

Secondly, an extension to arbitrary $q$ of the polynomials $\Psi_{\pi}$ was proposed in [16] and reformulated as a solution of the $q \mathrm{KZ}$ equation in [17]. The present work being entirely based on recurrence relations which are still satisfied by solutions of the $q \mathrm{KZ}$ equation, it is clear that it can be extended to generic $q$. This might shed some light on a possible generalization of the RS conjecture to arbitrary $q$. It would also allow to connect to the geometry of certain orbital varieties [17].

\section{Acknowledgments}

The author would like to thank A. Knutson and B. Nienhuis for discussions, and J.-B. Zuber for carefully rereading the manuscript. The author acknowledges support 
from European Marie Curie Research Training Networks "ENIGMA" MRT-CT-20045652, "ENRAGE" MRTN-CT-2004-005616, and from ANR program "GIMP" ANR-05BLAN-0029-01.

\section{Appendix A. Proof of symmetry under interchange of spectral parameters}

The purpose of this appendix is to provide an explicit proof of the symmetry of the partition function of weighted plane partitions when one switches the spectral parameters of two neighboring parallel lines, say $\gamma_{1}$ and $\gamma_{2}$. The weighting is the same as described in Sec. 3 (i.e. for simplicity we absorb powers of $q$ in the definition of the spectral parameters). The plane partitions are supposed to fill a domain with arbitrary shape; in fact, we shall only need to consider the region in which the two spectral parameters act and ignore the rest of the tiling.

Generally, this region has the shape of Fig. 8. Note that the positions of the triangular holes are in principle not fixed: to a given shape of the entire domain to be filled may correspond several possibilities of locations for these holes. However here we shall keep them fixed and show that each term in the summation over them is a symmetric function of $\gamma_{1}$ and $\gamma_{2}$. The latter is equivalent to showing that a certain family of row-to-row transfer matrices is commutative; however here, to avoid any formalism we shall prove this by explicit computation.

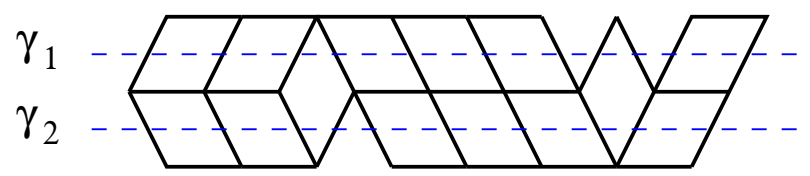

Fig. 8: Region of a tiling where the two spectral parameters $\gamma_{1}, \gamma_{2}$ appear.

The locations of the triangular holes are strongly constrained in order to allow the possibility of a lozenge tiling. As a consequence we see that we can divide our region into pieces separated by holes, so that these pieces have no influence on each other, and the summation over plane partitions factorizes accordingly. There are two types of pieces:

a. Hexagons of size $k \times 1 \times 1, k \geq 0$. They are those that are delimited either (i) on at least one side by a pair of adjacent holes (by parity on the other side the boundary must have the same shape) or (ii) by two individual holes, with the parity being such that there are forced edges going inwards on both sides. The weighted enumeration of plane partitions inside a hexagon of size $k \times 1 \times 1$ can be computed, cf Sec. 3:

$$
\frac{\prod_{i=1}^{k+1}\left(\alpha_{i}-\gamma_{1}\right)\left(\beta_{i}-\gamma_{2}\right)-\prod_{i=1}^{k+1}\left(\alpha_{i}-\gamma_{2}\right)\left(\beta_{i}-\gamma_{1}\right)}{\gamma_{1}-\gamma_{2}}
$$

where the $\alpha_{i}$ and $\beta_{i}$ are the spectral parameters in the other 2 directions. Eq. (A.1) is explicitly symmetric in $\gamma_{1}, \gamma_{2}$. 
b. Parallelograms $2 \times k, k \geq 1$ : they are those that are delimited by two individual holes on opposite boundaries, with the parity being such that there are forced edges going outwards. A parallelogram can only be filled by elementary lozenges in a unique way, hence the weight

$$
\prod_{i=1}^{k}\left(\alpha_{i}-\gamma_{1}\right)\left(\alpha_{i}-\gamma_{2}\right)
$$

where the $\alpha_{i}$ are the spectral parameters going parallel to the non-horizontal sides of the parallelogram.

We finally conclude that the whole partition function, being a sum (over the locations of holes) of $\gamma_{1}, \gamma_{2}$ independent functions (the partition function outside the region considered above) times products of functions of the type (A.1) or (A.2), is symmetric in $\gamma_{1}, \gamma_{2}$.

\section{References}

[1] A.V. Razumov and Yu.G. Stroganov, Combinatorial nature of ground state vector of O(1) loop model, Theor. Math. Phys. 138 (2004) 333-337; Teor. Mat. Fiz. 138 (2004) 395-400, math. C0/0104216.

[2] P. A. Pearce, V. Rittenberg and J. de Gier, Critical $Q=1$ Potts Model and Temperley-Lieb Stochastic Processes, cond-mat/0108051.

[3] A.V. Razumov and Yu.G. Stroganov, O(1) loop model with different boundary conditions and symmetry classes of alternating-sign matrices, Theor. Math. Phys. 142 (2005) 237-243; Teor. Mat. Fiz. 142 (2005) 284-292, cond-mat/0108103.

[4] P. A. Pearce, V. Rittenberg, J. de Gier and B. Nienhuis, Temperley-Lieb Stochastic Processes, J. Phys. A 35 (2002) L661-L668, math-ph/0209017.

[5] J. de Gier, Loops, matchings and alternating-sign matrices, math. C0/0211285.

[6] S. Mitra and B. Nienhuis, Osculating random walks on cylinders, in Discrete random walks, DRW'03, C. Banderier and C. Krattenthaler edrs, Discrete Mathematics and Computer Science Proceedings AC (2003) 259-264, math-ph/0312036.

[7] S. Mitra, B. Nienhuis, J. de Gier and M.T. Batchelor, Exact expressions for correlations in the ground state of the dense O(1) loop model, JSTAT (2004) P09010, cond-math/0401245.

[8] P. Di Francesco, A refined Razumov-Stroganov conjecture I: J. Stat. Mech. P08009 (2004), cond-mat/0407477; II: J. Stat. Mech. P11004 (2004), cond-mat/0409576.

[9] P. Di Francesco and P. Zinn-Justin, Around the Razumov-Stroganov conjecture: proof of a multi-parameter sum rule, Elec. J. Combinatorics 12 (1) (2005), R6, math-ph/0410061. 
[10] M.T. Batchelor, J. de Gier and B. Nienhuis, The quantum symmetric XXZ chain at $\Delta=-1 / 2$, alternating sign matrices and plane partitions, J. Phys. A34 (2001) L265-L270, cond-mat/0101385.

[11] P. Di Francesco, P. Zinn-Justin and J.-B. Zuber, A Bijection between classes of Fully Packed Loops and Plane Partitions, E. J. Combi. 11(1) (2004), R64, math. C0/0311220.

[12] B. Lindström, On the vector representations of induced matroids, Bull. London Math. Soc. 5 (1973) 85-90;

I. M. Gessel and X. Viennot, Binomial determinants, paths and hook formulae, Adv. Math. 58 (1985) 300-321.

[13] V. Korepin, Calculation of norms of Bethe wave functions, Comm. Math. Phys. 86 (1982) 391-418.

[14] P. Di Francesco, P. Zinn-Justin and J.-B. Zuber, Determinant Formulae for some Tiling Problems and Application to Fully Packed Loops, Annales de l'Institut Fourier 55 (6) (2005), 2025-2050, math-ph/0410002.

[15] F. Caselli and C. Krattenthaler, Proof of two conjectures of Zuber on fully packed loop configurations, J. Combin. Theory Ser. A 108 (2004), 123-146, math. CO/0312217.

[16] V. Pasquier, Quantum incompressibility and Razumov Stroganov type conjectures, cond-mat/0506075.

[17] P. Di Francesco and P. Zinn-Justin, Quantum Knizhnik-Zamolodchikov equation, generalized Razumov-Stroganov sum rules and extended Joseph polynomials, J. Phys. A 38 (2005) L815-L822, math-ph/0508059. 